\title{
Disseminação do conhecimento e da inovação através de diferentes formas de transferência de tecnologias aos produtores rurais
}

Dissemination of knowledge and innovation through different forms of technology transfer to rural producers

Difusión de conocimientos e innovación a través de diferentes formas de transferencia de tecnología a los productores rurales

Debora Zumkeller Sabonaro

ORCID: https://orcid.org/0000-0001-8604-7712 Universidade Federal de São Carlos, Brasil

E-mail: dzsabonaro@hotmail.com Luiz Antonio Martinelli ORCID: https://orcid.org/0000-0002-7103-7551 Universidade de São Paulo, Brasil E-mail: lamartinelli50@gmail.com

Janaina Braga do Carmo

ORCID: https://orcid.org/0000-0002-2871-9880 Universidade Federal de São Carlos, Brasil E-mail: jbcarmo2008@gmail.com

\begin{abstract}
Resumo
Este estudo objetiva identificar meios de transferir a tecnologia e a inovação tecnológica que contribuem para o desenvolvimento dos produtores rurais. A proposta está sendo desenvolvida pelo Programa de Pós-graduação em Biotecnologia e Monitoramento Ambiental, Universidade Federal de São Carlos, Campus Sorocaba, Estado de São Paulo- Brasil. Os procedimentos utilizados, neste trabalho, se caracterizam como interdisciplinar, bibliográfico, qualitativo, descritivo e estudo de múltiplo de casos. Foram analisadas a transferência de tecnologia através de eventos, geração de patentes para domínio público, geração de produto e registro de softwares para fins de transferência através de treinamento técnico e contrato de transferência de tecnologias aos produtores rurais realizados no Projeto: "Gestão de tecnologias geradas em projetos de expansão da cana-de-açúcar e seus efeitos ambientais" contando com o apoio da Fundação de Amparo à Pesquisa do Estado de São Paulo no Processo: 2017/18918-5. Através dos resultados obtidos, pode-se inferir que estas práticas são eficazes, principalmente através de meios digitais, aos produtores rurais, o que afirma a necessidade de incentivar estes processos de transferência de tecnologia. Além disso, este artigo demonstra como os pesquisadores podem maximizar a produção beneficiando a sociedade.
\end{abstract}

Palavras-chave: Produtos; Software; Eventos; Transferência de tecnologia; Sustentabilidade.

\begin{abstract}
This study aims to identify ways to transfer technology and technological innovation that contribute to the development of rural producers. The proposal is being developed by the Postgraduate Program in Biotechnology and Environmental Monitoring, Federal University of São Carlos, Campus Sorocaba, State of São Paulo, Brazil. The procedures used in this work are characterized as interdisciplinary, bibliographical, qualitative, descriptive and multiple case studies. Technology transfer through events, generation of patents for public domain, product generation and software registration were analyzed for transfer purposes through technical training and technology transfer contract to rural producers carried out in the Project: "Management of technologies generated in sugarcane expansion projects and their environmental effects" counting on the support of the São Paulo State Research Support Foundation in Process: 2017/18918-5. Through the results obtained, it can be inferred that these practices are effective, mainly through digital media, to rural producers, which affirms the need to encourage these technology transfer processes. Furthermore, this article demonstrates how researchers can maximize production benefiting society.
\end{abstract}

Keywords: Products; Software; Events; Technology transfer; Sustainability. 


\begin{abstract}
Resumen
Este estudio tiene como objetivo identificar formas de transferencia de tecnología e innovación tecnológica que contribuyan al desarrollo de los productores rurales. La propuesta está siendo desarrollada por el Programa de Postgrado en Biotecnología y Monitoreo Ambiental de la Universidad Federal de São Carlos, Campus Sorocaba, Estado de São Paulo, Brasil. Los procedimientos utilizados en este trabajo se caracterizan por ser interdisciplinarios, bibliográficos, cualitativos, descriptivos y de estudio de casos múltiples. Se analizó la transferencia de tecnología a través de eventos, generación de patentes de dominio público, generación de productos y registro de software con fines de transferencia a través de la capacitación técnica y el contrato de transferencia de tecnología a productores rurales realizado en el Proyecto: "Gestión de tecnologías generadas en proyectos de expansión de la caña de azúcar y sus efectos ambientales" contando con el apoyo de la Fundación de Apoyo a la Investigación del Estado de São Paulo en el Proceso: 2017/18918-5. A través de los resultados obtenidos, se puede inferir que estas prácticas son efectivas, principalmente a través de los medios digitales, para los productores rurales, lo que afirma la necesidad de fomentar estos procesos de transferencia de tecnología. Además, este artículo demuestra cómo los investigadores pueden maximizar la producción en beneficio de la sociedad.
\end{abstract}

Palabras clave: Productos; Software; Eventos; Transferencia de tecnología; Sostenibilidad.

\title{
1. Introdução
}

Inovações podem ser gerados em universidades e em centros de pesquisas a nível nacional e internacional. No entanto, esta tecnologia, desenvolvida com verbas públicas, atinge o receptor ou usuário da tecnologia desenvolvida. Podemos correlacionar inovação ambiental como um, processo, produto melhorando os benefícios ambientais (Johnstone et al., 2011).

O crescimento da produção agrícola também está relacionado à inovação. Assim a inovação pode ajudar a enriquecer o campo e dar maior valor ao investimento em tecnologia (Mendes \& Buainain, 2015).

A transferência de tecnologia (TT) está intimamente relacionada à geração de conhecimento (P\&D), portanto ambas as áreas fazem parte do processo de inovação tecnológica, ou seja, existe uma interface claramente definida entre elas (Balbino; Martinez; Galerani, 2011).

Balbino, Martinez e Galerani (2011) apontam que ao se considerar o processo de TT, existem alguns fatores não técnicos que são inerentes ao processo e limitam sua eficiência, como participantes ou irão participar; convergência institucional; métodos de TT e avaliação de eficiência; e discutir as necessidades ou avaliação das necessidades com as partes interessadas relevantes. Esta integração deve incluir a formação contínua de multiplicadores com base nas necessidades de exploração e nas considerações dos participantes relevantes, bem como a avaliação dos processos e atividades utilizadas no TT (Barbino, Barcelos, Stone, 2011).

A integração entre pesquisadores, professores, técnicos, empresários e produtores rurais podem produzir inovações, sendo importante diversas formas de TT objetivando o aumento da transferência, tanto de forma teórica quanto prática (Cordeiro et al., 2015). Segundo Brescia et al., 2016, a transferência de tecnologia são fundamentais para a divulgação.

A transferência de tecnologia é importante para gerar inovações, uma forma é a divulgação de informações, sendo fundamental para a tomada de decisão no meio rural (Sabonaro \& Braga 2020). Além disso, a gerar inovações com sustentabilidade é evidente no nosso dia a dia, buscando novos desafios (Sabonaro et al., 2019).

Segundo Bozeman et al. (2015) a transferência de tecnologia pode ser traduzida considerando tanto os agentes transferidor quanto o receptor. Para que ocorra a transferência de tecnologia o agente transferidor deve proporcionar formas de viabilidade do processo (Berbegal et al., 2015; Bozeman et al., 2015; Cavalcante et al. 2019). Uma das prováveis causas da existência é uma prática que pesquisadores possuem em transferir o que especificam em tecnologias. Neste contexto, este artigo contempla um estudo de caso com objetivo identificar a meios de transferir a tecnologia e a inovação tecnológica que contribuem para o desenvolvimento dos produtores rurais. 


\section{Metodologia}

A pesquisa científica seguiu um procedimento metodológico a fim de alcançar o objetivo proposto no estudo, sendo adotados foram a abordagem de pesquisa qualitativa, onde explanou por meio de um estudo múltiplo de casos, o aprofundamento da utilização do conhecimento e da inovação tecnológica como fator de desenvolvimento. A pesquisa ainda se classifica como descritiva, pois para que se chegue num resultado, foi necessário caracterizar o ambiente agrícola. Considerando a amplitude do estudo, o uso do estudo bibliográfico de natureza interdisciplinar. Após a compreensão do tema por meio de leituras de artigos, livros, dissertações e teses foi feito um levantamento das principais formas de transferência de tecnologias, visando auxiliar futuros projetos nesta linha.

Com relação à coleta de dados, o estudo de caso é um dos métodos mais completos, pois, este se obtem de fonte de dados de pessoas como também de dados documentais. Os estudos de caso podem derivar de um único, como de múltiplos casos (Gil, 2002).

As principais formas de transferência de tecnologias aplicadas no Projeto: "Gestão de tecnologias geradas em projetos de expansão da cana-de-açúcar e seus efeitos ambientais" contando com o apoio da Fundação de Amparo à Pesquisa do Estado de São Paulo no Processo: 2017/18918-5 foram através de eventos, geração de patentes para domínio público, geração de produto, geração e registro de softwares para fins de transferência através de treinamento técnico e contratos e transferência de tecnologia aos produtores rurais.

\section{Resultados}

\subsection{Eventos}

Os eventos agropecuários são fundamentais para os produtores rurais, pesquisadores, dentre outros. As informações tecnológicas produzidas nestes eventos são fundamentais e necessitam de meios eficientes de difusão, uma vez que os participantes destes eventos não representam a totalidade das pessoas interessadas por vários motivos como dificuldades de transporte, divulgação, e dificuldades ainda maiores devido à Pandemia que afetou mundialmente os eventos presenciais.

Os eventos 'Workshop de Inovação e transferência de tecnologia', 'Workshop de Inovações Interdisciplinares para Sustentabilidade' e 'Simpósio de Biotecnologia e Engenharia Florestal da UFSCar' foram eventos realizados presencialmente. Já o evento 'Treinamento Técnico do Software VegetaSolo (VS) foi realizado presencialmente e também contará com uma versão através de live com disponibilidade via internet. Os minicursos: 'Introdução a propriedade intelectual com foco em patentes' e 'Busca de anterioridades em Tecnologia' foram realizados via google meet.

\subsection{1 'Workshop de Inovação e Transferência de Tecnologia'}

Este evento contou com uma Mesa Redonda: 'Transferência de conhecimento e experiências adquiridas em campo para geração de tecnologias' e três apresentações orais com diferentes profissionais. Além das palestras, a uma mesa redonda foi fundamental, pois gerou maior integração entre os participantes do evento científico "Workshop de Inovação e Transferência de Tecnologia" ocorrido no Campus da UFSCAR Sorocaba, SP.

\subsection{2 'Workshop de Inovações Interdisciplinares para Sustentabilidade'}

O evento contou com uma Mesa Redonda: 'Projetos Tecnológicos: Tecnologia e transferência de tecnologia para Inovação' e uma apresentação oral da Palestra: ‘A pesquisa científica e/ou tecnológica na Empresa dentro do Programa de PósGraduação em Biotecnologia e Monitoramento Ambiental', além de apresentações de estudos de casos. 


\subsection{3 'Simpósio de Biotecnologia e Engenharia Florestal da UFSCar'}

Neste evento foram apresentadas duas palestras: 'A importância da pesquisa para geração e transferência de novas tecnologias' e 'Uso de técnicas de nucleação em áreas urbanas e rurais'; treinamento técnico na área de Levantamento Topográfico e contou ainda com uma mesa redonda: "A importância da pesquisa para geração e transferência de novas tecnologias" (Figura1).

Figura 1: 'Simpósio de Biotecnologia e Engenharia Florestal da UFSCar'.

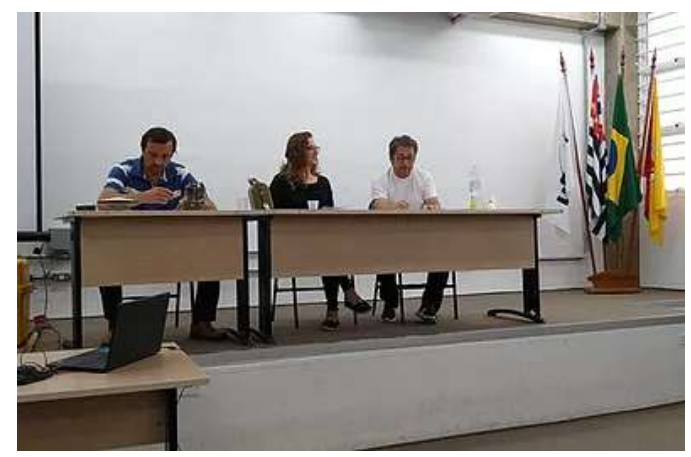

Fonte: Autores.

\subsubsection{Treinamento Técnico do Software VS}

A tecnologia foi desenvolvida para orientar os produtores rurais sobre o estado de conservação/ degradação de áreas de preservação permanente e ou/ áreas de nascentes utilizando tecnologia multiplataforma. Foi organizado um evento para ser realizado de forma presencial e será realizado também um evento através de live, com disponibilidade via internet, objetivando garantia da eficácia de divulgação.

\subsection{5 'Introdução a propriedade intelectual com foco em patentes' e 'Busca de anterioridades em Tecnologia'}

Foram realizados via google meet. Atingiu um público mediano por conta da falta de divulgação e por não ficar disponível na internet.

\subsection{Software- Desenvolvimento, registro e Transferência de Tecnologia}

O "Software para monitoramento do solo e água em áreas de preservação permanente e nascentes" foi realizado para auxiliar o diagnóstico sobre o estado de conservação de solos em nascentes, buscando transferir a tecnologia aos produtores rurais. Na elaboração do modelo foram considerados estudos sobre diagnósticos e programas de recuperação em nascentes. $\mathrm{O}$ conceito global utilizado neste modelo foi baseado em literatura e em estudos no campo sobre a caracterização de áreas ao longo de cursos d'água e no entorno de nascentes.

O software VegetaSolo pode ser empregado durante a fase de condução das atividades de recuperação propostas e de monitoramento ambiental. O App (aplicativo) VegetaSolo foi desenvolvido para orientar o produtor rural sobre o estado de conservação/ degradação de áreas de preservação permanente e ou/ áreas de nascentes utilizando tecnologia multiplataforma, sua flexibilidade de uso torna o software autointuitivo. O aplicativo está sendo testado em áreas de preservação permanente no Projeto: "Consequências ambientais da conversão pastagem-cana-de-açúcar e intensificação de pastagens" (2015/18790-3). Pode-se verificar que foi possível a utilização da metodologia aplicada para o desenvolvimento do software de fácil manuseio, facilitando a integração do produtor rural com a tecnologia aplicada. 


\subsubsection{Produto 1: Aplicativo}

O App (aplicativo) 'VegetaSolo' foi desenvolvido para orientar os produtores rurais sobre o estado de conservação/ degradação de áreas de preservação permanente e ou/ áreas de nascentes utilizando tecnologia multiplataforma, sua flexibilidade de uso torna o software autointuitivo.

O equipamento escolhido para a realização do Produto 1 foi o computador portátil Texas Nspire CX CAS. Este equipamento apresenta um ambiente de programação dedicado, bem como bibliotecas de programação para acesso global e funções e programas definidos pelo usuário. O software foi desenvolvido inicialmente em linguagem TBasic na IDE (TI-Nspire CX CAS versão Aluno v5.1) e transferido via USB para o computador portátil (Figura 2).

Figura 2: TBasic na IDE (TI-Nspire CX CAS versão v5.1).

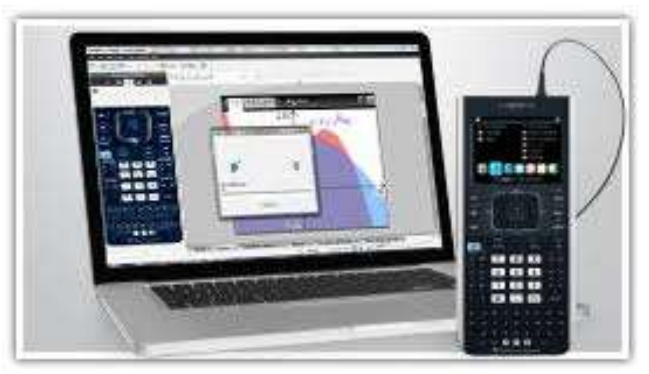

Fonte: Autores.

\subsubsection{Produto 2: Aplicativo "VegetaSolo"}

O Produto 2 está em fase de desenvolvimento e se caracteriza por ser um aplicativo (APP) para mobiles, inicialmente para Androide e IOS, disponível para o produtor rural, que poderá adquirir nas lojas de aplicativos.

Dotado de um software amigável, este aplicativo está sendo preparado para orientar os produtores rurais sobre o estado de conservação/ degradação de áreas de preservação permanente e ou/ áreas de nascentes utilizando tecnologia multiplataforma, sua flexibilidade de uso torna o software autointuitivo.

O banco de dados em nuvem, torna o aplicativo mais eficiente, podendo ser atualizados muito rapidamente fazendo do software uma ferramenta de informações e orientações constantes ao produtor rural. Este app será transferido aos pequenos e médios produtores rurais após término de um contrato de Transferência de Tecnologia entre a UFSCAR e a proprietária do software e a realização de Treinamento técnico será a etapa seguinte.

\subsection{Aplicação da Transferência de Tecnologia}

O Processo de Transferência de Tecnologia do Software para monitoramento do solo e água em áreas de preservação permanente e nascentes (VegetaSolo) está sendo realizado com apoio da Agência de Inovação da Ufscar nos anos de 2021 e 2022. Foram discutidas as formas que poderiam ser realizada a Transferência de Tecnologia. Para tanto a primeira etapa do trabalho foi a montagem de um Plano de Negócios. A próxima etapa foi escrever um contrato com normas, regras, prazos e formas de execução da transferência de Tecnologia. Um dos processos em andamento que faz parte da transferência é o treinamento técnico direcionada ao público beneficiário (produtores rurais). Este treinamento ensina como utilizar o Software e ainda, caso produtor tiver qualquer dúvida, ele terá um suporte ténico através de um canal de comunicação disponibilizado no aplicativo. 
O contrato de transferência de tecnologia está sendo realizado através de um projeto de extensão com a Universidade Federal de São Carlos. Este contrato poderia ser realizado entre Universidade Federal de São Carlos e uma empresa, mas para isto precisaria de um CNPJ.

\section{4 "Sonda Termográfica para monitoramento sustentável em campo"}

O processo de patenteamento da "Sonda Termográfica para Monitoramento Sustentável em campo" (INPI: BR 10 2020 020843) foi realizado a fim de se tornar domínio publico. Desta forma serão beneficiados os produtores rurais e demais públicos que tiverem interesse. Uma invenção com pedido de patente da forma domínio público pode ser comercializada e replicada (INPI, 2021).

A presente patente de invenção tem por objetivo auxiliar a análise de temperatura do solo. Essa forma de desenvolvimento da sonda termográfica vem solucionar inconvenientes como a difícil interpretação de leitura, a fragilidade à ação da natureza, a preocupação com o término da carga de baterias durante uma jornada de leitura. Em alguns casos, a difícil manipulação e transporte em campo, devido à quantidade de acessórios. Por outro lado, a surpresa de baterias baixas, ou descarregadas em campo está solucionada com a inserção de um pequeno painel solar, associado a uma bateria recarregável.

A Cabeça de Série da Sonda Termográfica para monitoramento sustentável em campo foi desenvolvida para atender as necessidades dos Produtores Rurais e atendimento da linha de Pesquisa do Projeto Temático. A Figura 3D representa o gabinete. O desenvolvimento do gabinete no Solidedge Studant versão 2021 está representado na Figura 3B. A Figura 3C mostra o gabinete sendo preparado para fatiamento no Ultimeker Cura para ser produzido na impressora 3D. A Figura 3D exibe a montagem em camadas do gabinete na impressora 3D Tronxy. Está exposto na Figura 3E o protótipo da sonda em campo com a placa solar.

Figura 3: Montagem da cabeça de série da Sonda Termográfica.

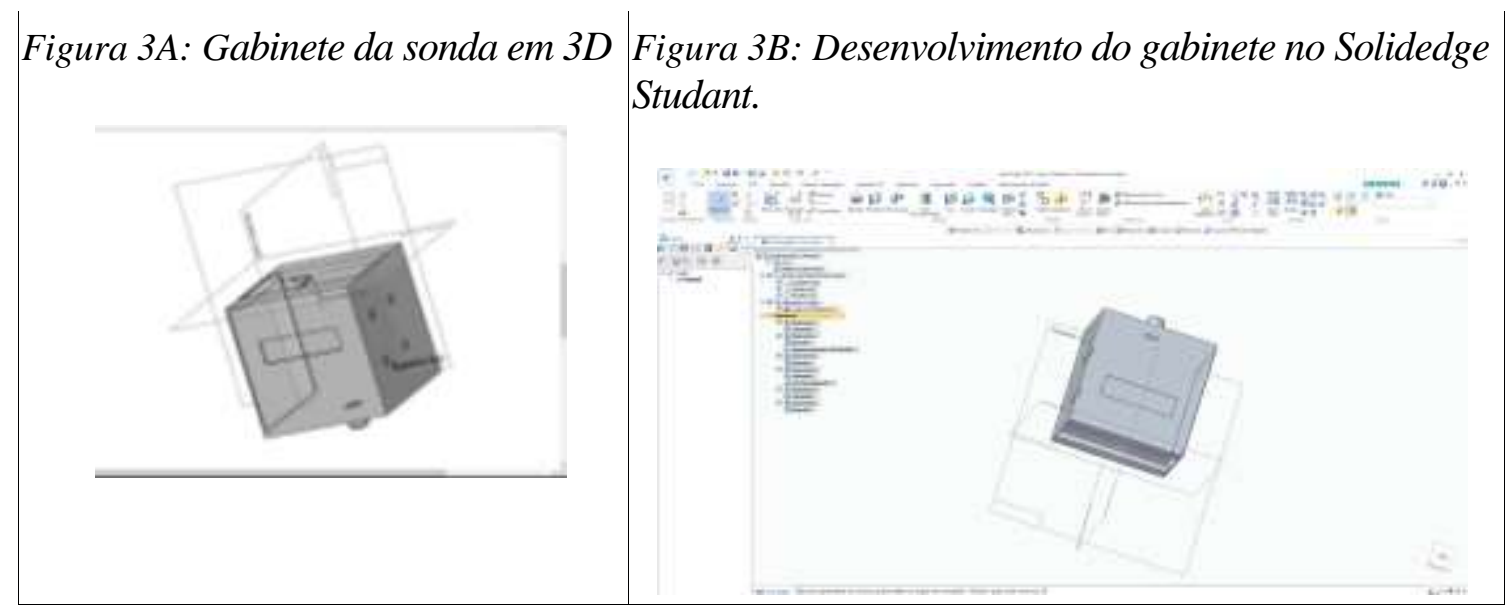




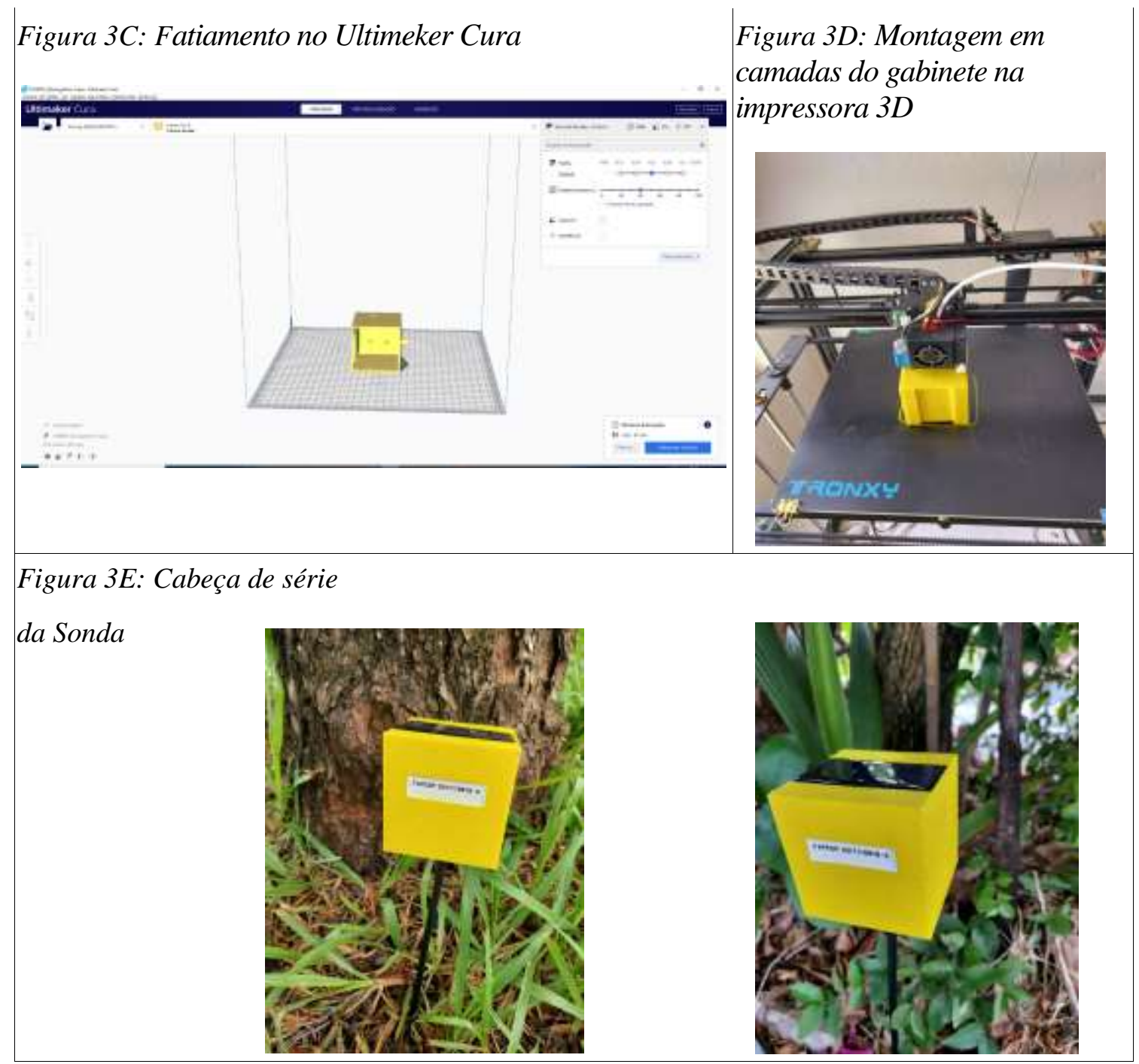

Fonte: Autores.

\subsubsection{Divulgação de Tecnologias}

O impacto das inovações a favor da modernização e difusão da tecnologia agricultura, é claramente visível. O uso da tecnologia digital sustenta o desenvolvimento de atividades cotidianas como cultivo, colheita e manejo de animais, insumos que atendam a necessidades específicas, softwares e aplicativos de gerenciamento de ativos, entre outras atividades. Além de transferência de tecnologia em eventos de tecnologia.

Hernández, Domínguez e Restrepo (2009) aplicam o monitoramento de tecnologia para destacar os conceitos e fundamentos das ciências no desenvolvimento e gestão de tecnologia. Portanto, verifica-se que se reflete principalmente em aplicações de produção inteligente, gestão e evolução dos processos de produção.

Os métodos de coleta são derivados principalmente da biometria, como teste científico, medição web e medição de patente, que diferem no objeto do estudo, mas são todos métodos quantitativos (Noronha; Maricato, 2008). Segundo Rivero; Sánchez; Suárez (2009) uma vez que são caracterizados por métodos quantitativos, eles são aplicados em estudos de tendências.

Silvina (2018) sugeriu em seu estudo que o uso de um aplicativo para smartphone, entre seus diversos recursos, ampliando a aplicabilidade das tecnologias produzidas e disseminadas durante os eventos. Com isso, segue a tendência de 
digitalização do setor, reduz a dificuldade de monitoramento de ocorrências de produtores rurais e auxilia os Órgãos Estaduais de Pesquisa Agronômica a finalizarem as premissas de fomento à sua agricultura.

As lives sofreram alterações principalmente com a Pandemia. No entanto, com a agragação de conteúdos acadêmicos, palestras, aulas e reuniões foram incorporadas no cotidiano das pessoas de forma eficiente e segura (Fettermann et al., 2020).

Observamos que, embora bem distribuídos, não se limitando à divulgação via site, os três primeiros eventos são voltados para o público da região. Cursos de treinamento de curta duração oferecidos por meio de reuniões com o público-alvo, mas não eram eficazes. A formação técnica, por outro lado, não se limitará ao grande público da região, mas funcionará como ferramenta de formação e divulgação e exploração de software.

\subsubsection{Transferência de Tecnologia}

Em um estudo de Quonion et al. (2014) a propriedade intelectual, especialmente patentes vem desempenhando um papel fundamental na área estratégica, como a transferência de tecnologia contribuindo com pesquisas sobre responsabilidade social e de valorização dos recursos naturais. As patentes são consideradas meios de expressão da pura pesquisa técnica e tecnológica do mercado. É claro que as patentes podem fazer pesquisas além de uma investigação técnica rigorosa (Jin, 2005).

A nomenclatura dos contratos de transferência pode sofrer variações. Um exemplo é o Código de Conduta de 16 de maio de 2013 , bem como no $\S 1^{\circ}$ do artigo $2^{\circ}$ da Lei $10.168 / 2000$.

Intervenções no campo econômico por royalties, contratos de transferência de tecnologia e os contratos relacionados são classificados principalmente de acordo com o objeto e a finalidade do registro, divididos em quatro tipos de contratos.

As ferramentas e fatos que viabilizam e implementam os processos de TT são: dias de campo, possibilitando o aprendizado pelo contato direto e facilitando assim a troca de conhecimentos entre técnicos e produtores; Cursos de formação voltados para a formação de pessoas engajadas no planejamento, organização e execução de atividades teóricas e práticas; participar de feiras e eventos: pesquisas para criação de oportunidades de negócios e troca de informações; Unidade de Demonstração (DU) e Unidade de Referência de Tecnologia (URT): Uma unidade de demonstração de tecnologia, sistemas e produtos, onde o DU não se preocupa com a coleta sistemática de informações sobre o desenvolvimento do sistema implantado, geralmente configurado para demonstração em um determinado evento; e exibição de tecnologia: espaço de exposição interativo (Balbino et al., 2015).

Na Transferência de Tecnologia, o trabalho conjunto é um meio eficiente, até porque ajudará na solução de eventuais litígios que possam surgir durante a vigência do contrato, da forma mais eficaz, no sentido de que os colaboradores estão atentos para fazer novos contratos e manter relações econômicas estáveis.

Neste projeto, o contrato de transferência de tecnologia beneficiará os produtores rurais e a Autoridade de Inovação. Assim, foram realizadas reuniões para definir os passos a serem tomados, uma das principais reuniões foi para fazer um plano de negócios. Com esse plano concluído, fica mais fácil dar os próximos passos, como a parte escrita do contrato.

\section{Considerações Finais}

A tecnologia proporcionou oportunidades, sendo necessária adaptações, principalmente com a pandemia. Uma das formas de promover o melhor acesso dos produtores rurais à informação tecnológica é a divulgação e realização de eventos pela Internet.

As ferramentas digitais de campo contribuem para o desenvolvimento social, econômico e ambiental em diversos campos. 
Durante a pandemia, os eventos na Internet aumentaram dramaticamente e tomaram forma e provavelmente continuarão a aumentar a fim de construir e reestruturar o conhecimento e melhorar as habilidades na implementação da transferência.

Observamos que embora bem distribuídos, não se limitando à divulgação do site das Universidades, os três primeiros eventos são voltados para o público da região. Os minicursos são realizados por meio de reuniões para um público seleto, mas carecem de publicidade em sua eficácia. A formação técnica, por outro lado, não se limitará ao grande público da região, além de funcionar como ferramenta de formação, divulgação e exploração de software.

A cooperação é importante para os contratos de Transferência de Tecnologia adequado para todas as partes envolvidas e também para limitar atitudes oportunistas, no sentido de que os colaboradores estão atentos para fazer novos contratos e manter relações econômicas estáveis.

Apesar das dificuldades enfrentadas pelas instituições, as ações das Agências de Inovação têm trabalhado para atender à necessidade crescente de proteção e transferência maduras. Neste projeto, o contrato de transferência de tecnologia beneficiará tanto os produtores rurais quanto a Agência de Inovação. Assim, foram realizadas reuniões para definir os passos a serem tomados, uma das principais reuniões foi para fazer um plano de negócios. Com esse plano concluído, fica mais fácil dar os próximos passos, como a parte escrita do contrato.

\section{Agradecimentos}

À Fundação de Amparo à Pesquisa do Estado de São Paulo- Processos 2017/18918-5 e 2015/18790-3.

\section{Referências}

Balbino, L. C.; Barcellos, A. O.; Stone, L. F. (Ed.) (2011). Marco Referencial: Marco Referencial: Integração Lavoura- Pecuária-floresta. Brasília, DF: Embrapa, 130 p.

Berbegal-Mirabent J, García, J. L. S., \& Ribeiro-Soriano, D. E. (2015). University-industry partnerships for the provision of R\&D services. Journal of Business Research. 15 (68), 1407-1413. doi: 10.1016/j.jbusres.2015.01.023

Bozeman, B.; Rimes, H., Youtie, J. (2015). The evolving state-of-the-art in technology transfer research: revisiting the contingent effectiveness model. Research Policy. 15(44), 34-49. doi: 10.1016/j.respol.2014.06.008

Brasil. Lei de Inovação Federal no 10.973, de 02 de dezembro de 2004. < http://www.mct.gov.br/index.php/content/view/8477.html

Brasil. Lei de Propriedade Industrial no 9279, de 14 de maio de 1996. < http://www.planalto.gov.br/ccivil_03/leis/19279.htm

Brescia, F., Colombo, G. \& Landoni, P. (2016). Organizational structures of knowledge transfer offices: an analysis of the world's top-ranked universities. The Journal of Technology Transfer. 16 (41): 132-151. doi: 10.1007/s10961-014-9384-5

Cavalcante, F. V.; Almeida, M. B. C. De; Renault, T. B. (2019). Intervenientes dos processos de transferência tecnológica em uma instituição de ciência e tecnologia: O Caso Fiocruz. Revista Gestão \& Tecnologia. $19 \quad$ (2), 217-239. <http://revistagt.fpl.edu.br/get/article/view/1383>. doi:https://doi.org/10.20397/2177-6652/2019.v19i2.1383.

Cordeiro, L. A. M.; Balbino, L. C.; Galerani, P. R; Domit, L. A.; Silva, P. C.; Kluthcouski, J.; Vilela, L.; Marchão, R. L.; Skorupa, L. A.; Wruck, F. J. (2015). Transferência de tecnologias para adoção da estratégia de integração Lavoura-Pecuária-Floresta. In: CORDEIRO, L. A. M.; Vilela, L; Kluthcouski, J.; Marchão, R. L. (Ed.). Integração Lavoura-Pecuária-Floresta: o produtor pergunta, a Embrapa responde. Brasília, DF: Embrapa, 377-393. (Coleção 500 Perguntas, 500 Respostas).

Fettermann, J. V., Benevenuti, C. B., Tamariz, A. D. R. (2020). Letramentos em processo: lives como um gênero textual acadêmico a partir da pandemia do COVID-19. Anais do Encontro Virtual de Documentação em Software Livre e Congresso Internacional de Linguagem e Tecnologia Online, [S.1.], 9 (1), 11-21. <http://www.periodicos.letras.ufmg.br/index.php/anais_linguagem_tecnologia/article/view/17696/1125613809>.

Gil, AC (2002). Como elaborar projetos de pesquisas. São Paulo: Atlas.

Hernández, C. N. J.; Domínguez, O. F. C..; Restrepo, L. A. M. (2009). Manufactura biológica e inteligente: atributos de la vida aplicados al desarrollo tecnológico. Revista Ingeniería e Investigación, 29 (2), 127-134.

Jin, Z. (2005). Global Technological Change: From Hard Technology to Soft Technology. Intellect: UK. p. 317, ISBN: 1841501247, janvier. 
Research, Society and Development, v. 11, n. 1, e1811123589, 2022

(CC BY 4.0) | ISSN 2525-3409 | DOI: http://dx.doi.org/10.33448/rsd-v11i1.23589

Johnstone, N., Haščič, I., \& Watson, F. (2011). Methodological issues in the Development of Indicators of Innovation and Transfer in Environmental Technologies. Invention and Transfer of Environmental Technologies, OCDE, Paris, 191-212. http://www.oecd.org/env/consumptioninnovation/48818512.pdf

Mendes, Cic \& Buainain, A. M. (2015). Transferência de Tecnologia: Análise além das fronteiras da Embrapa. https://ainfo.cnptia.embrapa.br/digital/bitstream/item/131728/1/tecnologiainformacao.pdf

Noronha, D. P. \& Maricato, J. M. (2008). Estudos métricos da informação: primeiras aproximações. Revista Eletrônica de Biblioteconomia e Ciências da Informação, 13 (1), 116-128.

Quoniam, L; Kniess, C. T.; Mazieri, M. R. (2014). A patente como objeto de pesquisa em Ciências da Informação e Comunicação Encontros Bibli: Revista eletrônica de biblioteconomia e ciência da informação, 19 (39), 243-268.

Rivero, Y. M.; Sánchez, M. V. G..; Suárez, Y. M. (2009). Modelo de evaluación para software que emplean indicadores métricos en la vigilancia científicotecnológica. ACIMED, 20 (6), 125-140.

Sabonaro, D. Z., Sabonaro, C. Z., Carmo, J. B. (2019). Renovabio. A certificação, tecnologia e transferência de tecnologia na produção de cana-de-acúcar com sustentabilidade. R. gest. sust. Ambien., Florianópolis. 2019; 8 (3), 725-735.

Sabonaro, D. Z. \& Braga, J. B. A. (2020). Transferência de tecnologia para o produtor rural: um caminho para a sustentabilidade. R. gest. sust. ambient., 9 (n. esp), 120-132.

Silvina, B. S. (2018). Modelo de Vigilância Tecnológica de Eventos Agropecuários para promoção da Transferência de Tecnologias, 147f. Tese apresentada como requisito parcial à obtenção do título de Doutora em Engenharia de Produção, do Programa de Pós-Graduação em Engenharia e Produção, da Universidade Tecnológica Federal do Paraná. 